Voix et Images

volxetimages

\title{
Approches et situation de l'essai québécois
}

\section{André Belleau}

Volume 5, numéro 3, printemps 1980

\section{Fernand Ouellette}

URI : https://id.erudit.org/iderudit/200231ar

DOI : https://doi.org/10.7202/200231ar

Aller au sommaire du numéro

\section{Éditeur(s)}

Les Presses de l'Université du Québec

\section{ISSN}

0318-9201 (imprimé)

1705-933X (numérique)

Découvrir la revue

\section{Citer cet article}

Belleau, A. (1980). Approches et situation de l'essai québécois. Voix et Images, 5(3), 537-543. https://doi.org/10.7202/200231ar d'utilisation que vous pouvez consulter en ligne.

https://apropos.erudit.org/fr/usagers/politique-dutilisation/ 


\section{Approches et situation de l'essai québécois}

Ces remarques portent sur certaines des questions et difficultés qu'implique l'étude de l'essai dans la littérature québécoise. Elles ne concernent pas tel ou tel essayiste ou écrivain mais l'essai à la fois comme discours et comme pratique historiquement et socialement situés. On a souvent dit que l'essai fait figure de parent pauvre dans notre littérature ${ }^{1}$, aussi bien du point de vue du nombre des textes que de l'intérêt qu'on leur a prêté. En revanche, l'essai en général, en tant que type de forme ou "genre", a fait l'objet d'analyses substantielles comme en font foi, entre autres, les deux ensembles publiés par la revue Etudes littéraires, le premier intitulé “L'Essai”2, le second, "Le Pamphlet"3, ainsi que l'ouvrage de Jean Terrasse: Rhétorique de l'essai littéraire ${ }^{4}$.

Je grouperai mes observations sous deux interrogations principales. En premier lieu, quel type d'approche aurait la plus grande valeur heuristique, en d'autres termes, serait la plus à même de favoriser la recherche: repérage des textes, délimitation des corpus, l'analyse elle-même? En second lieu, puisque les discours ne sont pas des phénomènes isolés mais font partie, à chaque moment historique, du système littéraire général, comment voir, toujours pour la littérature québécoise, les rapports possibles entre l'essai et d'autres discours tel le roman? Cette dernière question suppose que soit convoquée l'institution littéraire, entendue ici dans son sens généralement reçu comme l'ensemble formé d'une part par des faits d'appareil (l'école, la critique, l'édition, etc.), et d'autre part par une seconde série de faits qui sont euxmêmes des faits de discours ou plus précisément de code: les règles de production et de lecture des textes littéraires.

On gagnerait à ne pas s'imaginer qu'un quelconque secteur de ce que Marc Argenot a appelé la "prose d'idées" 5 pût échapper en principe à la littérature. En affirmant cela, je me rends bien compte que je vais, en apparence du moins, à contre-courant; on m'opposera la théorie jakobsonnienne des fonctions du langage de même que la tradition rhétorique et 
les recherches actuelles en ce domaine. Mais Jakobson n'est pas du tout aussi simple que d'aucuns le laissent souvent croire par l'usage un peu rapide qu'ils en font. II ne propose pas une typologie des discours littéraires et c'est donc une tendance bien fâcheuse, et fréquente, que celle qui consiste à hypostasier les fameuses fonctions linguistiques de manière à ce qu'elles définissent des formes ou des genres. La même remarque vaudrait d'ailleurs au sujet des neuf fonctions de Halliday ${ }^{6}$ ou des six "caractères spécifiques" du «style scientifique" selon Budagov?. Certes les travaux sur la rhétorique argumentative apparaissent indispensables dans la mesure où ils nous aident, lorsque nous retournons aux occurrences textuelles, à mesurer le degré de "réalisation" des codes, à rapporter les lacunes et les traits atypiques à des contraintes diverses dont par exemple la pression du discours social sur les codes rhétoriques. On admettra toutefois que la description engagée par des affirmations comme celle-ci: l'essai appartient au genre enthymématique et ainsi de suite, a peut-être comme limite de ne pas insérer l'essai dans un ensemble plus large avec les éléments duquel il entrerait en relation. Je reconnais que je me trouve ici sur un terrain délicat où j'avance, d'ailleurs, avec quelque difficulté. Tout se passe comme si le recours à un certain type d'étude rhétorique signalait une réelle difficulté à envisager l'essai selon les normes dominantes du littéraire. Or affirmer: tout n'est pas littéraire mais tout PEUT être littéraire, ce n'est pas seulement renoncer à la notion d'un prétendu langage neutre, adéquat, transparent, langage cognitif, scientifique ou quotidien, selon les besoins, sur le fond duquel le discours littéraire se mesurerait en termes d'une sorte d'intensité plus grande, c'est aussi impliquer un déplacement des critères du statique au fonctionnel, du typique au relationnel. II y a dans l'immobilité typologique, selon Frederic Jameson, comme la trace “d'une pensée qui, en mouvement vers l'histoire concrète, prend peur et cherche à transformer ses points de vue en essences éternelles... " 8

Pourquoi le nier? Je suis en train de donner un semblant lou un minimum) de cohérence démonstrative à des pensées et des intuitions qui me sollicitaient depuis un certain temps et qui ont été singulièrement activées il y a peu par deux remarques: l'une de Roland Barthes, l'autre de ce brillant essayiste qu'est Jean Marcel.

Dans le numéro qui lui a consacré la revue Tel Quel, Barthes déclare ce qui suit au sujet des Problèmes de linguistique générale d'Émile Benveniste: «[...] de tous les linguistes que j'ai lus, c'est Benveniste qui reste pour mai au plus haut rang [...] il y a a la surface de sa linguistique comme le frémissement, poignant à force d'être discret, d'une eau qui va bouillir : cette force, cette chaleur qui soulève la science (la plus rigoureuse qui soit, dans le cas de Benveniste) vers autre chose, est, vous le savez, ce que j'appelle l'écriture $"$ ?.

Cette observation de Barthes a évidemment un aspect paradoxal: comment peut-on parler, comme s'il s'agissait de littérature, du discours théoriquement et j'allais dire officiellement le moins littéraire possible, celui de la linguistique, de toutes les sciences humaines celle dont on dit qu'elle fut la première à se constituer en une science véritable? Mais ce paradoxe n'est qu'apparent. Impliquer que toute entreprise discursive, quelle qu'elle soit, est 
menacée par l'écriture, peut succomber à la tentation de l'écriture, c'est dire

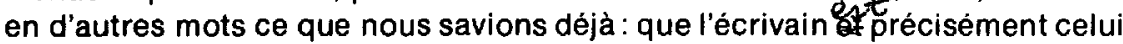
qui "travaille" le discours social, en principe toutes ses variétés, et que le discours scientifique, à l'instar du disburs journalistique ou du discours publicitaire, fait partie du discours social, c'est-à-dire de l'ensemble des messages qui nous parviennent et qui constituent notre environnement linguistique et idéologique. Mais il y a plus: en recouvrant métadiscursivement de métaphores le texte scientifique de Benveniste, Barthes se trouve, de façon négative, à contester ce qu'il faut bien appeler l'influence exorbitante du métadiscours poétique sur le système littéraire et sur l'institution. On constate en effet que le langage théorique et critique sur la poésie de même que ce que les poètes ne cessent de dire de leurs pratiques a fini par constituer le canon même de la littérarité en général. Les marques attribuées au discours poétique conçu erronément comme la réalisation la plus complète et la plus pure de la littérature se sont vues annexées, donc déjà occupées, donc rendues inutilisables pour d'autres types de discours. J'en donne comme exemple l'espèce d'équivalence qui a fini par s'instaurer entre langage poétique et langage métaphorique, avec l'exclusion de tout ce qui relèverait du narratif, du cognitif, et même du sémantisme. Une des raisons de l'embarras que nous éprouvons devant l'essai réside pour une bonne part dans l'inflation et la généralisation du métadiscours poétique dans le système et l'institution. Par quel bout le prendre en effet puisqu'à mesure que nous semblons nous éloigner du poétique, nous nous éloignons en même temps de la littérature elle-même?

Ajoutons par ailleurs que s'il semble y avoir accord de principe sur le fait que l'essai procède du fictionnel à l'instar de tout discours littéraire ${ }^{10}$, il existe encore peu d'études qui en tirent résolument les conséquences et envisagent l'essai - "roman sans noms propres "12 selon les mots de Barthes ou "biographie [...] sans événements»12 d'après ceux de Jean Marcel du point de vue narratif, c'est-à-dire d'une sorte de récit idéel comportant à sa manière sujets et fonctions. Un exemple fort probant serait l'analyse par Bernard Andrès du schéma actantiel dans les Pamphlets de Valdombre ${ }^{13}$. J'ai tenté pour ma part de montrer dans un texte ${ }^{14}$ hélas! insuffisamment développé comment cette fictionnalisation opère dans Un Génocide en douce de Pierre Vadeboncœur: ce que nous pensions être des adversaires politiques y deviennent les ennemis mêmes de la fable, leurs démarches et leurs attitudes acquièrent l'accent tragique du destin, les idées, les objets intellectuels, comme des sortes d'actants, s'affrontent en des combats dont les épisodes constituent une curieuse (et abstraite) trame idéologique.

Quelles conclusions provisoires conviendrait-il de tirer de ces premières considérations? D'abord, vu la configuration actuelle du discours théorique et critique, faisons l'hypothèse que l'essai dans la littérature québécoise n'est pas toujours là où on le cherche, que sa pratique, faute d'une caution spécifique, s'est même parfois déguisée pour se rapprocher davantage du feu littéraire central allumé par l'institution. Ensuite, formons le 
vœu qu'il soit possible d'élaborer graduellement ce que Marc Angenot a appelé une "essayistique "15, mais non pas fondée en majeure partie sur une reprise de la rhétorique aristotélicienne ou classique. II serait souhaitable, à mon avis, que l'essayistique contribuât à un réaménagement du discours théorique et critique institutionnel de façon à ce que la littérarité ne soit plus définie principalement par la poésie (et à un moindre degré le roman), ceci, non pas en s'attachant à quelques caractéristiques ignorées jusqu'ici, mais en investissant à ses propres fins les critères existants déjà réquisitionnés et devenus de ce fait plus ou moins exclusifs: la métaphorisation et la figuralité en général, les propriétés du signifiant, les ruptures, etc. Je me plais à rêver qu'un jour, on dira l'Essayistique et non le Poétique pour désigner ce qu'il y a de littéraire dans tous les discours; cela, certes, ferait de la peine à bien des braves gens mais serait plus en conformité avec le système actuel des genres dans leurs situations réelles.

Pour revenir une dernière fois à la fonction métaphorique, elle ne se limite certes pas pour l'essai à servir l'argumentation. Dans un article remarquable ${ }^{16}$, Judith Schlanger a éclairé le rôle essentiel de la métaphore dans la littérature réflexive: outil de recherche, de connaissance qui ouvre et explore les chemins du "pensable", fait avancer le discours, permet à ceux que l'auteur nomme "les artistes de la discursivité abstraite»17 de nous donner, selon ses propres termes, des "spectacles discursifs"18 et des " paysages du sens $" 19 .$. On retrouve par ce biais, les aperçus de Barthes, qui parle d'un “chant d'idées-phrases »20, et pour lequel « tout essai repose [...] sur une vision des objets intellectuels" 21 , objets soumis non pas "à une instance de vérité mais à une pensée des effets $" 22$. L'objet intellectuel, ajoute Barthes, se crée par une sorte de pesée sur le signifiant „23. Se dessine ici, me semble-t-il, un espace de travail où un discours critique (et même esthétique) sur l'essai aurait profit à s'exercer et à se déployer. Quant à l'essai considéré comme une "fiction idéelle", voilà un parti qu'il serait intéressant, après l'avoir pris, de pousser jusqu'au bout. Le seul fait de se demander si Novalis est aussi fictif dans Fernand Ouellette que Napoléon dans Stendhal ouvre de fascinantes perspectives.

J'en arrive à la seconde des "remarques" dont j'ai parlé plus haut, et qui m'ont guidé dans cet essai sur l'essai : la remarque de Jean Marcel. La voici : "[...] l'essai est la forme caractérisée de l'introduction dans le discours littéraire du JE comme générateur d'une réflexion de type lyrique sur un corpus culturel agissant comme médiateur entre les tensions fragmentées de l'individualité dans sa relation à elle-même et au monde" 24 . Un peu plus loin, Jean Marcel précise: «L'individualité semble ne plus pouvoir se comprendre elle-même qu'à travers son insertion dans la série historique et culturelle qui l'a produite» 25 . Je ne sais trop en fait s'il s'agit là d'une "remarque», d'une définition, d'une hypothèse, d'une description, d'une observation, peu importe; en revanche, ce que je n'ignore pas, c'est que ces paroles ont le même pouvoir connecteur et par là opératoire que la vision lukacsienne du héros de roman : voici l'essai branché sur un énonciateur, sur un état et un statut possibles du langage et de la culture, et par eux, sur l'ensemble des 
codes sociaux. Rien ici qui préjuge d'une structure formelle. Et ce "je" posé comme signalant sa position dans le monde des signes de la culture ne doit pas être confondu, est-il besoin de le dire, avec un "je" hors-texte. II est luimême un produit du langage à l'instar du narrateur dans un roman. Mais tandis que dans le roman, le je-héros se situe par rapport aux événements, aux personnes, à la structure, à l'évolution d'une société fictive, le “je" de l'essai s'éprouve dans un espace (et un temps) tout aussi fictif composé d'objets culturels ou plutôt de signes culturels. Cette conception aide à mieux comprendre la position de l'essai dans la littérature québécoise. Pendant longtemps, notre roman nous a été donné comme se racontant tout seul, sans retour réflexif de l'énonciateur sur son énoncé au sujet des codes littéraires, de la langue, etc. L'essai, au contraire, implique ce distancement car il nous propose précisément du langage sur du langage, de la culture sur de la culture, des signes sur des signes. II ne cherche pas dans la culture horstexte ce qui doit en être dit, mais il trouve et développe dans le langage ce qui peut être dit de la culture. Une société dont le ou les langages sont cuturellement peu saturés ne favorise donc pas la production d'essais. Attribuer à la censure cléricale la relative pénurie d'essais dans la littérature québécoise demeure une vue superficielle des choses. Une des recherches valables - toujours dans cette avenue ouverte par Jean Marcel - consisterait à évaluer le degré d'intégration des objets culturels - appelons-les références culturelles - à la discursivité textuelle. Elles peuvent rester extérieures à la diégèse idéelle, jouant le rôle d'indices ornementaux; mais il leur arrive aussi d'avoir une fonction dynamique dans le procès discursif. On est renvoyé, dans les deux cas, aux conditions concrètes de production du texte ainsi qu'au statut effectif du langage et de la culture dans la société réelle. Comparer les essais de Fernand Ouellette et de Pierre Vadebonccur serait à cet égard riche d'enseignement.

Me voici arrivé à la dernière partie de ce trop bref exposé, celle où je me proposais de voir un peu le rapport entre l'essai et d'autres discours dans le système littéraire québécois. Je retiens de tout ce qui précède que la question souvent posée: manquons-nous d'essayistes? devrait être remplacée par une autre: manquons-nous d'essais? D'une part, nous en avons fait l'hypothèse, l'essai ne se désigne pas toujours comme tel, et de l'autre, le choix par un écrivain de l'essai, du poème, du roman, dépend souvent de facteurs extérieurs à lui et liés au statut de telle ou telle forme dans l'institution littéraire 26. Or j'estime qu'au Québec, depuis au moins une quarantaine d'années, un bon nombre d'essais ont été produits et publiés sous le couvert du discours romanesque. Qu'on ne se méprenne pas: il ne s'agit pas des remarques, commentaires adjuvants qui constituent, dans tous les romans, le discours habituel du narrateur. Parlons plutôt de longs développements, ou de chapitres entiers, ou de fragments nombreux et disjoints, ou même d'une organisation narrative exclusivement centrée sur ce que Jean Marcel a 
appelé un "corpus culturel». Encore une fois, non pas de simples aplages" idéologiques mais de considérables expansions discursives.

Les Demi-civilisés de Jean-Charles Harvey offrent dès 1934 un bon exemple. Le premier chapitre à lui seul constitue tout un essai sans compter le reste. On sent dans Les Plouffe de Lemelin (1948) la tentation de l'essai (épisode de la grève à "L'Action chrétienne") mais cela tourne court. En revanche, Mon fils pourtant heureux de Jean Simard, publiée en 1956, renferme un traité sur les mœurs et sur l'éducation tandis que Le prix $d u$ Souvenir de Jean-marie Poirier (1957) réfléchit longuement sur la formation de l'écrivain et la nature de la littérature. Gérard Bessette publie en 1958 un roman curieux et attachant, La Bagarre, dans lequel sont discutées les conditions linguistiques et socioculturelles de la littérature. Aucune créature de Charbonneau, en 1961, donne le plus de place possible à des considérations sur la littérature et la politique. Mais il y a encore bien des exemples: La Défaillance, roman de Gilbert Choquette paru en 1969, peut très bien être reçu comme un essai sur la philosophie chrétienne. Et on pourrait aisément allonger cette liste avec le Ciel de Québec de Jacques Ferron, L'Antiphonaire d'Hubert Aquin, D'amour, P.Q. de Jacques Godbout, $L \theta$ Semestre de Gérard Bessette. Comme on s'en rend compte, ceci n'est pas négligeable. II semblerait que nous ayons plus d'essais que d'essayistes. II faudrait invoquer ici, je le répète, un discours social culturellement peu chargé qui rend la diction intellectuelle plus vraisemblable dans la fiction du roman que dans celle de l'essai. En d'autres termes, si toutes les sociétés se racontent des histoires, il en est où l'on parle rarement à table de littérature et de musique. Et il convient d'ajouter que cette prolifération de l'essai sur le corps romanesque a sans doute quelque rapport avec l'impossibilité dans laquelle s'est trouvé notre roman de faire place au discours réaliste, problème qui a été longuement étudié par Gilles Marcotte 27. Vues sous l'angle historique, ces contaminations ne paraissent guère étonnantes. Qu'on se souvienne de la poésie scientifique à la Renaissance ${ }^{28}$. La science, au $X \mid X^{6}$ siècle, est elle-même devenue roman comme l'ont montré pour la paléontologie Marc Angenot et Nadia Khouri dans un travail important à paraître bientôt. Et on pourra lire sur la « fictionnalisation" de la médecine Illness as Metaphor de Susan Sontag ${ }^{29}$.

Le système littéraire québécois présente toutefois un fait paradoxal de portée théorique peut-être notable. Si l'essai paraît plus proche du roman dans la mesure où ils ont en commun un procès discursif, la plupart de nos meilleurs essayistes actuels sont cependant des poètes. Je dis la plupart car le Gilles Marcotte du Roman à l'imparfait et le Pierre Vadeboncour des Deux Royaumes demeurent des essayistes considérables. Mais songez au Gaston Miron du Recours didactique, à Fernand Ouellette, à Pierre Trottier, Fernand Dumont, Jacques Brault, Madeleine Gagnon... Et j'allais oublier, mais c'était d'un autre temps, le Journal de Saint-Denys-Garneau. Théorème provisoire: quand les romanciers ont envie d'écrire des essais, ils écrivent des romans; quand les poètes ont envie d'écrire des essais, ils les écrivent. II se pourrait donc qu'il y eût entre la pratique de la poésie et celle de l'essai plus d'affinités ${ }^{30}$ que ne le laisserait supposer la rhétorique argumentative. Ma 
protestation contre l'impérialisme de la poésie a peut-être été injustifiée. Dans ce cas, mon texte tend à se défaire lui-même au lieu de conclure, ce qui est bien, après tout, la meilleure façon de conclure.

\section{André BELLEAU \\ Université du Québec à Montréal}

1. Voir la remarquable synthèse de François Ricard, “L'Essai», dans Études françaises, vol. 13, n०3-4, octobre 1977, notamment pp. 378-381. Cet article comporte de précieuses indications bibliographiques.

2. Vol.5, no 1 , avril 1972.

3. Vol. 11, no 2, août 1978.

4. Les Presses de l'Universitè du Québec, Collection "Genres et discours ", 1977.

5. Angenot, Marc, "La parole pamphlétaire, esquisses d'un cadre typologique", Etudes littéraires, vol. 11, no 2, août 1978, p. 255.

6. Voir Langue française, no 37 , février 1978, p. 42.

7. Rondeau, Guy, "Les langues de spécialité ", Le Français dans le monde, $n^{\circ} 145$, mai-juin 1979, p. 76.

8. Jameson, Frederic, Marxism and Form, Princeton University Press, 1974, pp. 93-94.

9. Barthes, Roland, "Réponses", dans $T e l$ Quel, no 47, automne 1971, p. 98.

10. Ecoutons François Ricard, loc. cit., p. 367: "Le langage de l'essai [...] est autant, sinon plus, "performatif" qu' informatif "; le signifiant y fait plus que transmettre le signifié, il l'invente, le modèle, agit constamment sur lui".

11. Roland Barthes par Roland Barthes, Seuil, 1975, p. 124.

12. Marcel, Jean, "Forme et fonction de l'essai dans la littérature espagnole", Études littéraires, vol. 5, no 1, avril 1972, p. 81.

13. Andres, Bernard, "Pour une grammaire de l'énonciation pamphlétaire", Etudes littéraires, vol. 11, no 2, août 1978, pp. 351-373.

14. "Un génocide en douce de P. Vadeboncour : un discours crépusculaire", Voix et Images, vol. III, no 1, septembre 1977, pp. 154-156.

15. Angenot, Marc, loc. cit., p. 255.

16. Schlanger, Judith, “Langage intellectuel, langage métaphorique», Littérature, no 29, février 1978, pp. 21-37.

17. Ibid., p. 21.

18. Ibid., p. 22.

19. Ibid., p. 37.

20. Roland Barthes par Roland Barthes, p. 111.

21. Ibid., p. 94.

22. Ibid., p. 94.

23. Ibid., p. 138.

24. Marcel, Jean, Ioc. cit., p. 87 - (Les soulignés sont dans le texte).

25. Ibid., p. 85.

26. Voir à ce sujet Jacques Dubọis, L'Institution de la littérature, Nathan/Labor, 1978 , p. 49 sq.

27. Marcotte, Gilles, Le Roman à l'imparfait, La Presse, 1976.

28. Schmidt, Albert-Marie, La Poésie scientifique en France au XV/e siècle, Editions Rencontre, 1970.

29. Farrar, Strauss et Giroux, 1978.

30. Ricard, François, loc. cit., p. 367 : “ [...] l'essai [...] appartient peut-être autant à l'ordre du poème qu'à celui du discours".

N.D.L.R.: Nous remercions M. Jean-Cléo Godin, directeur du centre d'études québécoises de l'Université de Montréal de nous avoir communiqué ces textes présentés lors d'un mini-colloque le 5 octobre 1979. 